\title{
Neumann heat flow and gradient flow for the entropy on non-convex domains
}

\author{
Janna Lierl, Karl-Theodor Sturm
}

\begin{abstract}
For large classes of non-convex subsets $Y$ in $\mathbb{R}^{n}$ or in Riemannian manifolds $(M, g)$ or in RCDspaces $(X, d, m)$ we prove that the gradient flow for the Boltzmann entropy on the restricted metric measure space $\left(Y, d_{Y}, m_{Y}\right)$ exists - despite the fact that the entropy is not semiconvex and coincides with the heat flow on $Y$ with Neumann boundary conditions.
\end{abstract}

\section{Introduction}

Throughout this paper, let $(X, d)$ be a complete locally compact geodesic space and let $m$ be a locally finite Borel measure with full topological support. We always assume that the metric measure space $(X, d, m)$ satisfies the $\operatorname{RCD}(K, \infty)$-condition for some finite number $K \in \mathbb{R}$. Recall that this means that the Boltzmann entropy w.r.t. $m$

$$
\text { Ent }_{m}: \mu \mapsto \begin{cases}\int f \log f d m, & \text { if } \mu=f m \\ \infty, & \text { if } \mu \nless m\end{cases}
$$

is weakly $K$-convex on the $L^{2}$-Wasserstein space $\left(\mathcal{P}_{2}(X), W_{2}\right)$ of probability measures on $(X, d)$ with finite second moments and that the Cheeger energy on $(X, d, m)$

$$
\mathrm{Ch}: f \mapsto \liminf _{\substack{g \rightarrow f i n L^{2}(X, m) \\ g \in \operatorname{Lip}(X, d)}} \int|\mathrm{D} g(x)|^{2} d m(x)
$$

is a quadratic functional on $L^{2}(X, m)$, cf. Section 4 .

It is well-known from the fundamental work of Ambrosio, Gigli, and Savaré AGS14a that, for all $f_{0} \in L^{2}(X, m)$ with $\mu_{0}=f_{0} m \in \mathcal{P}_{2}(X)$, the following are equivalent

- $t \mapsto f_{t}$ is a gradient flow for $\mathrm{Ch}$ in $L^{2}(X, m)$

- $t \mapsto \mu_{t}=f_{t} m$ is a gradient flow for $\operatorname{Ent}_{m}$ in $\left(\mathcal{P}_{2}(X), W_{2}\right)$.

For $X=\mathbb{R}^{n}$, this is the celebrated result of Jordan, Kinderlehrer, and Otto [JKO98. Since any closed convex subset $Y \subset X$ inherits the $\operatorname{RCD}(K, \infty)$-condition, the same equivalence holds for the heat flow on $Y$ which should be regarded as the 'heat flow on $Y$ with Neumann boundary conditions on $\partial Y^{\prime}$ '. For non-convex $Y$, however, such an equivalence seems to be unknown so far - even in the Euclidean case.

Here and in the sequel, 'gradient flow' will always be understood in the so-called EDE-sense. In the previous situation the equivalence holds true also in the stronger formulation of gradient flows in the $\mathrm{EVI}_{K}$-sense. In general, however, the $\operatorname{RCD}(K, \infty)$-condition does not hold for non-convex subsets $Y \subset X$, thus there cannot exist $\mathrm{EVI}_{K}$-gradient flows for the entropy.

Our main result is that - under slightly more restrictive assumptions on $(X, d, m)$ and under mild assumptions on $Y$ - there exists an (EDE-)gradient flow for the entropy and this flow necessarily coincides with the heat flow.

Theorem 1.1. Let $(X, d, m)$ be an $\operatorname{RCD}^{*}(K, N)$-space and $Y$ be a regularly $\kappa$-convex set (for some $\kappa \leq 0)$, where $K \in \mathbb{R}$ and $N>0$ finite. Then for all $f_{0} \in L^{2}\left(Y, m_{Y}\right)$ with $\mu_{0}=f_{0} m_{Y} \in$ $\mathcal{P}(Y)$ the following are equivalent 
(i) $t \mapsto f_{t}$ is a gradient flow for $\mathrm{Ch}_{Y}$ in $L^{2}\left(Y, m_{Y}\right)$

(ii) $t \mapsto \mu_{t}=f_{t} m_{Y}$ is a gradient flow for $\operatorname{Ent}_{m_{Y}}$ in $\left(\mathcal{P}(Y), W_{2, d_{Y}}\right)$.

The basic assumption here is that the set $Y \subset X$ is (regularly) $\kappa$-convex. It means that $Y$ can be represented as sublevel set of some ('regular') function $V: X \rightarrow \mathbb{R}$ which is $\kappa$-convex for some $\kappa \leq 0$. Our proof of Theorem 1.1 heavily depends on what we call the Convexification Theorem. It is the second main result of this paper and of independent interest.

Theorem 1.2. Let $Y$ be a locally $\kappa$-convex subset in an $R C D(K, \infty)$-space $(X, d, m)$. Then for every $\kappa^{\prime}<\kappa$ the set $Y$ is locally geodesically convex in the metric measure space $\left(X, d^{\prime}, m\right)$ where

$$
d^{\prime}(x, y):=\inf \left\{\int_{0}^{1} e^{-\kappa^{\prime} V\left(\gamma_{t}\right)}\left|\dot{\gamma}_{t}\right| d t: \quad \gamma:[0,1] \rightarrow X \text { abs. continuous, } \gamma_{0}=x, \gamma_{1}=y\right\} .
$$

In the case of regularly $\kappa$-convex sets, for appropriate choices of $V=V_{\varepsilon}$ the metric $d^{\prime}$ is uniformly equivalent to $d$ with ratio arbitrarily close to 1 . The convexity of $Y$ in $\left(X, d^{\prime}\right)$ will be proved using the contraction property

$$
d\left(x_{t}, y_{t}\right) \leq e^{-\kappa^{\prime} t} d\left(x_{0}, y_{0}\right)
$$

for the EVI-gradient flow for $V$ in $(X, d)$. Actually, the latter property will be extended to gradient flows for functions that are 'locally $\kappa$-convex' on some sets $Z \subset X$ which are not necessarily convex.

The discussion of $\kappa$-convex functions, $\kappa$-convex sets, and the Convexification Theorem will be the topic of Section 2. In Section 3, we prove that the convexification transform with regularly $\kappa$-convex potentials stays within the class of RCD-spaces. Section 4 is devoted to the study of the Cheeger energy $\mathrm{Ch}_{Y}$ on the restricted metric measure space $\left(Y, d_{Y}, m_{Y}\right)$. Among others, we prove that it coincides with the Neumann energy on $Y$ induced by Ch. Finally, we identify the gradient flows for $\mathrm{Ch}_{Y}$ in $L^{2}\left(Y, d_{Y}\right)$ with the gradient flows for $\operatorname{Ent}_{m_{Y}}$ in $\mathcal{P}_{2}\left(Y, d_{Y}\right)$.

\section{$1.1 \quad$ Some preliminaries}

\section{Gradients and gradient flows}

Let us recall some general notions. Let $\left(\mathbf{Y}, d_{\mathbf{Y}}\right)$ be a complete metric space. Let $E: \operatorname{Dom}(E) \rightarrow$ $(-\infty,+\infty]$ be a functional with domain $\operatorname{Dom}(E) \subset \mathbf{Y}$. The pointwise Lipschitz constant of $E$ is defined as

$$
|D E|(y):=\operatorname{lip} E(y):=\limsup _{x \rightarrow y, x \neq y} \frac{|E(y)-E(x)|}{d_{\mathbf{Y}}(x, y)}, \quad \text { if } y \in \operatorname{Dom}(E),
$$

$|D E|(y):=0$ if $y \in \operatorname{Dom}(E)$ is an isolated point, and $|D E|(y):=+\infty$ if $y \in \mathbf{Y} \backslash \operatorname{Dom}(E)$.

A function $g: \mathbf{Y} \rightarrow[0, \infty]$ is an upper gradient of $f: \mathbf{Y} \rightarrow[-\infty,+\infty]$ if for any curve $\gamma:[0,1] \rightarrow \mathbf{Y}$ that is absolutely continuous on the interval $(0,1)$, the map $s \mapsto g\left(\gamma_{s}\right)\left|\dot{\gamma}_{s}\right|$ is measurable in $[0,1]$ and $|f(\gamma(0))-f(\gamma(1))| \leq \int_{\gamma} g$.

Definition 1.3. Let $\left(\mathbf{Y}, d_{\mathbf{Y}}\right)$ be a metric space and let $E: \operatorname{Dom}(E) \rightarrow(-\infty,+\infty]$ be a functional with domain $\operatorname{Dom}(E) \subset \mathbf{Y}$. A gradient flow for $E$ in $\mathbf{Y}$ starting from $y_{0} \in \operatorname{Dom}(E)$ is a locally absolutely continuous curve $\left(y_{t}\right)_{t \in[0, \infty)} \subset \operatorname{Dom}(E)$ such that

$$
E\left(y_{0}\right)=E\left(y_{t}\right)+\frac{1}{2} \int_{0}^{t}\left|\dot{y}_{r}\right|^{2} d r+\frac{1}{2} \int_{0}^{t}\left|D^{-} E\right|^{2}\left(y_{r}\right) d r, \quad \forall t \geq 0 .
$$

Here the descending slope of $E$ is defined as

$$
\left|D^{-} E\right|(y):=\limsup _{x \rightarrow y, x \neq y} \frac{[E(y)-E(x)]_{+}}{d_{\mathbf{Y}}(x, y)}, \quad \text { if } y \in D(E),
$$

$\left|D^{-} E\right|(y):=0$ if $y \in \operatorname{Dom}(E)$ is an isolated point, and $\left|D^{-} E\right|(y):=+\infty$ if $y \in \mathbf{Y} \backslash \operatorname{Dom}(E)$. 
To distinguish these kind of gradient flows from other (related but not equivalent) ones they are also called gradient flows in the EDE-sense ('energy-dissipation equality').

\section{The curvature-dimension condition}

Let $(X, d, m)$ be a complete metric measure space and let $K, N \in \mathbb{R}$ with $N \geq 1$.

Definition 1.4. (i) We say that $(X, d, m)$ satisfies $\operatorname{CD}(K, \infty)$ if for any $\mu_{0}, \mu_{1} \in \mathcal{P}_{2}(X)$ with $W_{2}\left(\mu_{0}, \mu_{1}\right)<\infty$ there exists a (constant speed, as always) $W_{2}$-geodesic $\left(\mu_{t}\right)_{0 \leq t \leq 1}$ in $\mathcal{P}_{2}(X)$ between $\mu_{0}$ and $\mu_{1}$ satisfying

$$
\operatorname{Ent}_{m}\left(\mu_{t}\right) \leq t \operatorname{Ent}_{m}\left(\mu_{1}\right)+(1-t) \operatorname{Ent}_{m}\left(\mu_{0}\right)-\frac{1}{2} K t(1-t) W_{2}\left(\mu_{0}, \mu_{1}\right)^{2}
$$

('weak $K$-convexity of $\operatorname{Ent}_{m}$ on $\left(\mathcal{P}_{2}(X), W_{2}\right)$ '), Stu06, LV09.

(ii) If in addition to (i) the Cheeger energy $\mathrm{Ch}$ is a quadratic form on $L^{2}(X, m)$, then we say that $(X, d, m)$ satisfies $\operatorname{RCD}(K, \infty)$, AGS14b].

(iii) We say that $(X, d, m)$ satisfies $\mathrm{RCD}^{*}(K, N)$, if the Cheeger energy $\mathrm{Ch}$ is a quadratic form on $L^{2}(X, m)$ and $(X, d, m)$ satisfies $\mathrm{CD}^{*}(K, N)$ in the sense of [BS10] or, equivalently, $\mathrm{CD}^{e}(K, N)$ in the sense of [EKS15].

Remark 1.5. a) If $(X, d, m)$ satisfies $\operatorname{RCD}(K, \infty)$, then every $W_{2}$-geodesic $\left(\mu_{t}\right)_{0 \leq t \leq 1}$ in $\mathcal{P}_{2}(X)$ satisfies (2), see AGS14b, Proposition 2.23]. This property is called 'strong $\bar{K}$-convexity of $\operatorname{Ent}_{m}$ on $\left(\mathcal{P}_{2}(X), W_{2}\right)$ ' or simply 'strong $\mathrm{CD}(K, \infty)$ '.

b) The (strong) $\mathrm{CD}(K, \infty)$-condition (2) can be rephrased as the condition that for each $W_{2}$-geodesic $\left(\mu_{t}\right)_{0 \leq t \leq 1}$ the function $t \mapsto \operatorname{Ent}_{m}\left(\mu_{t}\right)$ is lower semicontinuous on [0,1], absolutely continuous on $(0,1)$ and satisfies

$$
\frac{\partial^{2}}{\partial t^{2}} \operatorname{Ent}_{m}\left(\mu_{t}\right) \geq K W_{2}\left(\mu_{0}, \mu_{1}\right)^{2}
$$

in distributional sense on $(0,1)$. The (strong) $\mathrm{CD}^{e}(K, N)$-condition is obtained by tightening up the latter to

$$
\frac{\partial^{2}}{\partial t^{2}} \operatorname{Ent}_{m}\left(\mu_{t}\right) \geq K W_{2}\left(\mu_{0}, \mu_{1}\right)^{2}+\frac{1}{N}\left(\frac{\partial}{\partial t} \operatorname{Ent}_{m}\left(\mu_{t}\right)\right)^{2} .
$$

For metric measure spaces which are essentially non-branching this is equivalent to the (strong) reduced curvature-dimension condition $\mathrm{CD}^{*}(K, N)$; see [EKS15] which provides many equivalent characterizations.

\section{Convexification}

The main result of this section will be the Convexification Theorem 2.17 Given a non-convex subset $Y$ in the geodesic space $(X, d)$, it provides a method to transform the metric $d$ into a conformally equivalent metric $d^{\prime}$ such that $Y$ is locally convex in $\left(X, d^{\prime}\right)$. We will be interested in a class of subsets that we call $\kappa$-convex sets with $\kappa \leq 0$. In the smooth Riemannian setting, these are precisely the sets with uniform lower bound $\kappa$ on the second fundamental form of $\partial Y$.

For the rest of this paper, we let $(X, d, m)$ be a complete locally compact geodesic metric measure space, and $m$ is locally finite Borel measure on $X$ with full support. In this section, we assume that $\operatorname{RCD}(K, \infty)$ is satisfied for some $K \in \mathbb{R}$. We let $\kappa \in \mathbb{R}$ be a real number (later on always $\kappa \leq 0)$ and $V: X \rightarrow(-\infty,+\infty]$ will be a lower bounded, continuous function (w.r.t. the topology of the extended real line). 


\subsection{Geodesically convex sets and $\kappa$-convex functions}

Definition 2.1. (i) A subset $Y \subset X$ is called convex if every geodesic $\left(\gamma_{s}\right)_{s \in[0,1]}$ in $X$ completely lies in $Y$ provided that $\gamma_{0}, \gamma_{1} \in Y$.

(ii) A subset $Y \subset X$ is called locally geodesically convex if there exists an open covering $\bigcup_{i} X_{i} \supset Y$ such that each geodesic $\left(\gamma_{s}\right)_{s \in[0,1]}$ in $X$ completely lies in $Y$ provided $\gamma_{0}, \gamma_{1} \in Y \cap X_{i}$ for some $i$.

Of course, the latter follows if the sets $Y \cap X_{i}$ are convex for each $i$. In general, neither $X_{i}$ nor $Y \cap X_{i}$ will be convex. Our definition tries to avoid any formulation based on coverings by convex subsets since for general geodesic spaces this is a delicate issue. Proving that small balls are convex requires an upper bound on the sectional curvature which is not at disposal for RCD-spaces and e.g. not true for the Grushin space.

Example 2.2. (i) For $\alpha \in(\pi / 2, \pi)$, the set $Y=[-\alpha, \alpha]$ is a locally geodesically convex (but not convex) subset of $X=S^{1}$ parametrized as $(-\pi, \pi]$.

(ii) The set $Y=\{(t, \phi): t \in \mathbb{R}, \phi \neq t(\bmod 2 \pi)\}$ is a locally geodesically convex (but not convex) subset of $X=\mathbb{R} \times S^{1}$.

Example 2.3. Let $(X, d)$ be the geodesic space induced by the Grushin operator

$$
L=\frac{\partial^{2}}{\partial x^{2}}+x^{2} \frac{\partial^{2}}{\partial y^{2}}
$$

on $X=\mathbb{R}^{2}$. Consider the unit-speed curve

$$
\varphi: t \mapsto\left(\sin (t), \frac{2 t-\sin (2 t)}{4}\right)
$$

which is locally a geodesic. Restricted to time intervals of length $\leq \pi$ it is a minimizing geodesic CL12. For instance, for each $k$ the restriction to $[k \pi,(k+1) \pi)]$ it is one of two possible minimizing geodesics connecting the points $\left(0, k \frac{\pi}{2}\right)$ and $\left(0,(k+1) \frac{\pi}{2}\right)$ - the other one is the curve $\left(-\varphi_{1}(t), \varphi_{2}(t)\right)$. Let $Y$ be the set on the right of the graph of $\varphi$, i.e.

$$
Y=\left\{(x, y): y=\frac{2 t-\sin (2 t)}{4} \Rightarrow x \geq \sin (t)\right\} .
$$

This set, of course, is not convex. For instance, the (unique) minimizing geodesic connecting the points $\left(-\frac{1}{2}, \frac{1}{4} \pi\right)$ and $\left(-\frac{1}{2}, \frac{3}{4} \pi\right)$ will not stay within $Y$.

But it is locally geodesically convex. Any covering by ('intrinsic') unit balls $X_{i}$ will do the job: each geodesic with endpoints in one of the sets $Y \cap X_{i}$ will stay in $Y$ (since $\varphi(s), \varphi(t) \in X_{i}$ implies $|t-s| \leq 2<\pi)$.

But neither $Y \cap X_{i}$ nor $X_{i}$ will be convex for unit balls centered at some $(0, y) \in Y$. For instance, if $X_{i}$ is the closed unit ball centered at the origin then the point $\varphi(1)$ and its mirror point $\left(-\sin (1), \frac{2-\sin (2)}{4}\right)$ will both be in $Y \cap X_{i}$ but their geodesic midpoint $\left(0, \frac{2-\sin (2)}{4}\right)$ is not in $X_{i}$.

Remark 2.4. In order to verify that a set $Y$ is locally geodesically convex it suffices that there exists an open covering $\bigcup_{i} X_{i} \supset \partial Y$ with the same property as above: each geodesic $\left(\gamma_{s}\right)_{s \in[0,1]}$ in $X$ completely lies in $Y$ provided $\gamma_{0}, \gamma_{1} \in Y \cap X_{i}$ for some $i$.

Proof. Let an open covering $\bigcup_{i} X_{i}$ of $\partial Y$ be given with the above property. Choose an open covering $\bigcup_{j} Z_{j}$ of $Y^{\prime}=Y \backslash \bigcup_{i} X_{i}$ by sets $Z_{j}$ whose diameter is smaller than their distance to $X \backslash Y$. Then of course any geodesic with endpoints in $Z_{j}$ cannot leave $Y$. Thus $\bigcup_{i} X_{i} \cup \bigcup_{j} Z_{j}$ is an open covering of $Y$ with the requested property.

Lemma 2.5 ([Stu, Corollary 2]). The following properties are equivalent: 
(i) $V$ is weakly $\kappa$-convex in the sense that for each $x_{0}, x_{1} \in X$, there exists a geodesic $\gamma$ : $[0,1] \rightarrow X$ from $x_{0}$ to $x_{1}$ such that

$$
V(\gamma(t)) \leq(1-t) V(\gamma(0))+t V(\gamma(1))-\frac{\kappa}{2} t(1-t)|\dot{\gamma}|^{2}, \quad \forall t \in[0,1] .
$$

(ii) $V$ is strongly $\kappa$-convex in the sense that (4) holds for all $x_{0}, x_{1} \in X$ and for every geodesic $\gamma:[0,1] \rightarrow X$ from $x_{0}$ to $x_{1}$.

(iii) $V$ is $\kappa$-convex in the EVI-sense: for each $x_{0} \in \overline{\{V<\infty\}}$ there exists an EVI $I_{\kappa}$-gradient flow for $V$ starting at $x_{0}$, that is, a locally absolutely continuous curve $\left(x_{t}\right)_{t>0}$ in $\{V<\infty\}$ with $\lim _{t \downarrow 0} x_{t}=x_{0}$ such that, for all $z \in X$ and a.e. $t>0$,

$$
\frac{1}{2} \frac{\partial}{\partial t} d^{2}\left(x_{t}, z\right) \leq-\frac{\kappa}{2} d^{2}\left(x_{t}, z\right)+V(z)-V\left(x_{t}\right) .
$$

Definition 2.6. (i) A lower bounded, continuous function $V: X \rightarrow(-\infty, \infty]$ will be called $\kappa$-convex on $X$ if it satisfies some/all properties of Lemma 2.5,

(ii) $V$ is called $\kappa$-convex on a closed subset $Z \subset X$ if there exists a covering $Z \subset \bigcup_{i} X_{i}$ by convex open sets $X_{i} \subset X$ such that each $\left.V\right|_{\overline{X_{i}}}: \bar{X}_{i} \rightarrow(-\infty, \infty]$ is $\kappa$-convex.

Proposition 2.7. Assume $Z \subset X$ is closed and that $V$ is finite and $\kappa$-convex on $Z$.

(i) For each $x_{0} \in Z$ there exist $\tau \in(0, \infty]$ and a locally absolutely continuous curve $\left(x_{t}\right)_{t \in[0, \tau)}$ in $X^{\prime}:=\bigcup_{i} X_{i}$ starting in $x_{0}$ with the property that for all $z \in X$ and a.e. $t>0$ such that $x_{t}$ and $z$ belong to a common set $X_{i}$ of the previous Definition 2.6(ii)

$$
\frac{1}{2} \frac{\partial}{\partial t} d^{2}\left(x_{t}, z\right) \leq-\frac{\kappa}{2} d^{2}\left(x_{t}, z\right)+V(z)-V\left(x_{t}\right)
$$

('local $E V I_{\kappa}$ gradient flow' for $V$ starting at $x_{0}$ ) with $\lim _{t \nearrow_{\tau}} x_{t}=x_{\tau} \in \partial X^{\prime} \subset X \backslash Z$ if $\tau<\infty$.

(ii) For any $x_{0}, y_{0} \in Z$, the associated local $E V I_{\kappa}$-gradient flows $\left(x_{t}\right)$ and $\left(y_{t}\right)$ satisfy the contraction property

$$
d\left(x_{t}, y_{t}\right) \leq e^{-\kappa t} d\left(x_{0}, y_{0}\right)
$$

for all $t \geq 0$ with the property that for each $s \in[0, t]$ a connecting geodesic for $x_{s}, y_{s}$ completely lies in $Z$.

Proof. (i) Let $\left(X_{i}\right)$ be a covering of $Z$ as in Definition 2.6(ii). For each $i$, the set $\bar{X}_{i}$ is closed and convex. Hence, the restriction of $d$ and $m$ to $X_{i}$ yields an $\operatorname{RCD}(K, \infty)$-space $\left(X_{i}, d_{i}, m_{i}\right)$. Indeed, optimal transport between measures in $\mathcal{P}_{2}\left(X_{i}\right)$ takes place along geodesics in $(X, d)$. By the convexity of $X_{i}$, all geodesics between points in $X_{i}$ completely lie in $X_{i}$. Hence, geodesics in $\left(\mathcal{P}_{2}(X), W_{2, d}\right)$ between measures in $P_{2}\left(X_{i}\right)$ are also geodesics in $\left(P_{2}\left(X_{i}\right), W_{2, d_{i}}\right)$.

On $\left(X_{i}, d_{i}, m_{i}\right)$, there exists a (unique) $\mathrm{EVI}_{\kappa}$-gradient flow for $V$, see [Stu, Theorem]. Uniqueness of $\mathrm{EVI}_{\kappa}$-gradient flows implies that the flows for $V$ on $X_{i}$ and on $X_{j}$ coincide on $X_{i} \cap X_{j}$. (For the uniqueness assertion, note that it suffices to verify (5) for all $z$ in a neighborhood of $x_{t}$.) Patching together the flows on the $X_{i}$ 's yields the 'local EVI ${ }_{\kappa}$-gradient flow' on $X^{\prime}=\bigcup_{i} X_{i}$ with life time $\tau$. Since $X^{\prime}$ is open, $\tau$ is non-zero.

(ii) Consider a time $s$ for which the floating points $x_{s}$ and $y_{s}$ can be joined by a geodesic $\left(\gamma_{r}^{s}\right)_{r \in[0,1]}$ in $(X, d)$ that completely lies in $Z$. Because each $X_{i}$ is convex, the geodesic $\left(\gamma^{s}\right)$ passes through each $X_{i}$ for one interval of times $r \in[0,1]$. Thus, we can find a finite number $n$ and $r_{k} \in[0,1]$ for $k=0,1, \ldots, n$ with $r_{0}=0, r_{n}=1$ such that, for each $k$, the points $\gamma_{r_{k}}^{s}$ and $\gamma_{r_{k+1}}^{s}$ lie in one of the sets $\left(X_{i}\right)$, say in $X_{i_{k, s}}$. The local EVI $\mathrm{EL}_{\kappa}$-gradient flows starting in these points will remain in $X_{i_{k, s}}$ at least for a short time, say for $s^{\prime} \in\left[s, s+\delta_{s}\right]$. Thus for all these $s^{\prime}$, according to [Stu, (3)],

$$
d\left(\gamma_{r_{k}}^{s^{\prime}}, \gamma_{r_{k+1}}^{s^{\prime}}\right) \leq e^{-\kappa\left(s^{\prime}-s\right)} d\left(\gamma_{r_{k}}^{s}, \gamma_{r_{k+1}}^{s}\right) .
$$

Adding up these distances for $k=0,1, \ldots, n-1$ yields

$$
d\left(\gamma_{0}^{s^{\prime}}, \gamma_{1}^{s^{\prime}}\right) \leq e^{-\kappa\left(s^{\prime}-s\right)} d\left(\gamma_{0}^{s}, \gamma_{r_{1}}^{s}\right) .
$$

This implies $\frac{\partial}{\partial s}\left(e^{\kappa s} d\left(\gamma_{0}^{s}, \gamma_{1}^{s}\right)\right) \leq 0$. Integrating over all $s \in[0, t]$ proves the assertion. 
Let $(\Delta, \operatorname{Dom}(\Delta))$ be the Laplacian on $(X, d, m)$. The space of test functions as defined in Gig, Definition 3.1.2] is

$$
\operatorname{TestF}(\mathrm{X}):=\left\{f \in \operatorname{Dom}(\Delta) \cap L^{\infty}(X): \Gamma(f) \in L^{\infty}, \Delta f \in W^{1,2}(X)\right\} .
$$

For definitions of $\Gamma, \Gamma_{2}$ and the Hessian, we refer to Section 4.1 .

Lemma 2.8. For $V \in \operatorname{TestF}(X)$ and for any $\kappa \in \mathbb{R}$ the following are equivalent

(i) $V$ is $\kappa$-convex.

(ii) $\mu \mapsto \int V d \mu$ is $\kappa$-convex on $\left(\mathcal{P}_{2}(X), W_{2}\right)$.

(iii) For all $f \in \operatorname{Dom}(\Delta)$ with $\Delta f \in W^{1,2}(X)$,

$$
\operatorname{Hess}_{V}(\nabla f, \nabla f) \geq \kappa \Gamma(f)
$$

holds in a weak sense, i.e. when integrated against any non-negative function $g \in \operatorname{Dom}(\Delta)$ with $g, \Delta g \in L^{\infty}(X)$.

Proof. This result - more precisely, the equivalence of (i) and (iii) - was already derived in Ket15, Theorem 7.2]. For convenience, we present an independent proof.

In view of [Vil09, Corollary 7.22], (i) $\Rightarrow$ (ii) is clear.

(ii) $\Rightarrow$ (i). Let points $x_{0}, x_{1} \in X$ be given. Let $\left(\mu_{t}\right)_{t}$ be a geodesic in $\left(\mathcal{P}_{2}(X), W_{2}\right)$ from $\delta_{x_{0}}$ to $\delta_{x_{1}}$. Each of the measures $\mu_{t}$ must be supported by $t$-midpoints of geodesics from $x_{0}$ and $x_{1}$. Choose one of these $t$-midpoints $\gamma_{t}$ with minimal $V\left(\gamma_{t}\right)$. Then

$$
V\left(\gamma_{t}\right) \leq \int V d \mu_{t} \leq(1-t) V\left(x_{0}\right)+t V\left(x_{1}\right)-\frac{\kappa}{2} t(1-t) d^{2}\left(x_{0}, x_{1}\right) .
$$

This proves the claim.

(ii) $\Rightarrow$ (iii). For $t \in(0, \infty)$ consider the weighted metric measure space $\left(X, d, e^{-t V} m\right)$ which satisfies a $\operatorname{RCD}\left(K+t^{\prime} \kappa\right)$-condition for any $t^{\prime}<t$ by Proposition 3.1. This implies a Bochner inequality for the associated weighted Dirichlet form,

$$
\Gamma_{2}^{t V} \geq(K+t \kappa) \Gamma^{t V}
$$

in a weak sense, see [AGS14b, Remark 6.3]. But $\Gamma_{2}^{t V}=\Gamma_{2}+t \cdot \operatorname{Hess}_{V}$ whereas $\Gamma^{t V}=\Gamma$. Dividing by $t$ and letting $t \rightarrow \infty$, this yields the claim

$$
\operatorname{Hess}_{V} \geq \kappa \Gamma
$$

(iii) $\Rightarrow$ (ii). Assuming the lower bound on the Hessian, we get

$$
\Gamma_{2}^{t V} \geq(K+t \kappa) \Gamma^{t V}
$$

for all $t \geq 0$. Thus again by the equivalence of the Eulerian and Lagrangian formulation of synthetic Ricci bounds AGS15, this yields the $\operatorname{RCD}(K+t \kappa)$-condition, hence strong $\operatorname{CD}(K+t \kappa)$ by Remark 1.5 for the weighted metric measure space $\left(X, d, e^{-t V} m\right)$, and thus the strong $\left(\frac{1}{t} K+\kappa\right)$-convexity of $\mu \mapsto \frac{1}{t} \operatorname{Ent}(\mu)+\int V d \mu$. In the limit as $t \rightarrow \infty$, this proves that the functional $\mu \mapsto \int V d \mu$ is strongly $\kappa$-convex on $\left(\mathcal{P}_{2}(X), W_{2}\right)$, that is, for any geodesic $\left(\mu_{t}\right)_{t \in[0,1]}$ in $\left(\mathcal{P}_{2}(X), W_{2}\right)$ and all $t \in[0,1]$,

$$
\int V d \mu_{t} \leq(1-t) \int V d \mu_{0}+t \int V d \mu_{1}-\frac{\kappa}{2} t(1-t) W_{2}\left(\mu_{0}, \mu_{1}\right)^{2} .
$$

(Firstly, this inequality will follow for all geodesics whose endpoints have finite entropy. An appoximation argument allows to get rid of this restriction.) 


\subsection{Locally $\kappa$-convex sets}

Definition 2.9. A subset $Y \subset X$ is called locally $\kappa$-convex if there exists a continuous function $V: X \rightarrow \mathbb{R}$ with $Y=\{x \in X: V(x) \leq 0\}$ and if for each $\delta>0$ there exists $r>0$ such that the function $V$ is $(\kappa-\delta)$-convex on $\overline{Y_{r}^{0}}$ with $|D V| \geq 1-\delta$ where $Y_{r}^{0}:=\{0<V \leq r\}$.

We will always assume that $m(Y)>0$.

Proposition 2.10. Let $X$ be a smooth Riemannian manifold, let $Y$ be the closure of a bounded, open subset of $X$ with smooth boundary, and fix $\kappa \leq 0$. Then the following are equivalent

(i) For each $\delta>0$ there is a function $V: X \rightarrow \mathbb{R}$ such that $Y=\{x \in X: V(x) \leq 0\}$, and there is a neighborhood $U$ of $\partial Y$ such that $V$ is smooth on $U, H e s s_{V} \geq \kappa-\delta$ on $U$, and $|D V| \geq 1-\delta$ on $U$.

(ii) For each $\kappa^{\prime}<\kappa$ there is a neighborhood $U$ of $\partial Y$ such that Hess $s_{V} \geq \kappa^{\prime}$ holds on $U$ with $V:= \pm d(., \partial Y)$ being the signed distance from the boundary.

(iii) The real-valued second fundamental form $\mathcal{I}_{\partial Y}$ satisfies $\mathcal{I}_{\partial Y} \geq \kappa$.

In the above proposition, $\mathcal{I}_{\partial Y}$ denotes the second fundamental form of $\partial Y$ in $Y$, defined as $\mathcal{I}_{\partial Y}(u, w):=\left\langle n, \widetilde{D}_{u} W\right\rangle n$, where $u, w \in T_{m} \partial Y, \widetilde{D}$ denotes the covariant derivative, $n$ is the inward unit normal vector at $m$, and $W$ is a vector field in a neighborhood of $m$, tangent to $\partial Y$ at any point of $\partial Y$ and with value $w$ at $m$.

Proof. (ii) $\Rightarrow$ (i): It follows from [AS96, Theorem 3.1] that the signed distance function is smooth in a neighborhood of $\partial Y$ and satisfies $|D V|=1$ on this neighborhood of $\partial Y$.

(i) $\Rightarrow$ (iii): For $z \in \partial Y$ and $\xi, \psi \in T_{z} \partial Y$,

$$
\mathcal{I}_{\partial Y}(\xi, \psi)=\frac{1}{|D V(z)|} \operatorname{Hess}_{V}(\xi, \psi)
$$

and thus

$$
\mathcal{I}_{\partial Y}(\xi, \xi) \geq \kappa|\xi|^{2}
$$

(iii) $\Rightarrow$ (ii): Choose $V:= \pm d(., \partial Y)$. Then for $z \in \partial Y$ and $\xi \in T_{z} \partial Y$,

$$
\operatorname{Hess}_{V}(\xi, \xi)=\mathcal{I}_{\partial Y}(\xi, \xi) \geq \kappa|\xi|^{2} .
$$

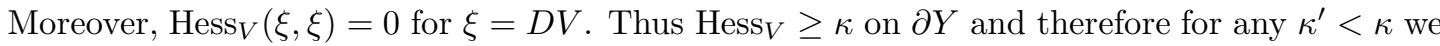
obtain $\operatorname{Hess}_{V} \geq \kappa^{\prime}$ on a suitable neighborhood of $\partial Y$.

Proposition 2.11. Assume that $(X, d)$ is an Alexandrov space with generalized sectional curvature $\geq L$. Let $r \in \mathbb{R}_{+}$with $r<\frac{\pi}{2 \sqrt{L}}$ if $L>0$ and arbitrary otherwise. Then $Y=X \backslash B_{r}(z)$, the complement of the ball with radius $r$ around $z \in X$, is locally $\kappa$-convex with

$$
-\kappa= \begin{cases}\frac{1}{r}, & \text { if } L=0 \\ \sqrt{L} \cot (\sqrt{L} r), & \text { if } L>0 \\ \sqrt{-L} \operatorname{coth}(\sqrt{L} r), & \text { if } L<0\end{cases}
$$

Proof. Put

$$
V_{0}(x)=\frac{1}{\Phi^{\prime}(r)}(\Phi(r)-\Phi(d(x, z))), \quad V(x)=\max \left\{V_{0}(x), 0\right\}
$$

where

$$
\Phi(r)= \begin{cases}\frac{1}{2} r^{2}, & \text { if } L=0 \\ -\frac{1}{L} \cos (\sqrt{L} r), & \text { if } L>0 \\ -\frac{1}{L} \cosh (\sqrt{-L} r), & \text { if } L<0\end{cases}
$$


Then $Y=\{V \leq 0\}$ and $\lim _{r \rightarrow 0} \inf _{x \in B_{r}(Y) \backslash Y}|D V(x)|=1$. Moreover,

$$
\text { Hess } V_{0}(x) \geq \frac{1}{\Phi^{\prime}(r)} \Phi^{\prime \prime}(d(x, z)) \cdot \operatorname{Id}
$$

for all $x \in X$, see e.g. AB03, Proposition 3.1]. (This inequality has to be understood in some weak sense. The precise meaning is in terms of suitable convexity along geodesic curves or more elementary - in terms of comparison of distances in corresponding triangles.) Thus, in particular,

$$
\operatorname{Hess} V_{0}(x) \geq \kappa \cdot \text { Id }
$$

with $\kappa$ as above for all $x \in B_{r}(z)$. The precise meaning here is exactly $\kappa$-convexity of $V_{0}$ in the sense of Lemma 2.5. Since $\kappa$-convexity is preserved by taking pointwise maximum, this therefore implies Hess $V(x) \geq \kappa \cdot$ Id for all $x \in X$.

Corollary 2.12. Let $(X, d)$ be an Alexandrov space with generalized sectional curvature $\geq L$ and as before let $r \in \mathbb{R}_{+}$with $r<\frac{\pi}{2 \sqrt{L}}$ if $L>0$ and arbitrary otherwise. Assume that $Y \subset X$ satisfies the exterior ball condition with radius $r$. That is,

$$
X \backslash Y=\bigcup_{x \in Z_{r}} B_{r}(x)
$$

with $Z_{r}:=\{z \in X: d(x, Y)>r\}$. Then $Y$ is locally $\kappa$-convex with $\kappa$ as above.

Proof. For each $z \in Z_{r}$ put

$$
V_{z}(x)=\frac{1}{\Phi^{\prime}(r)}(\Phi(r)-\Phi(d(x, z)))_{+}
$$

where $\Phi$ is as in (7). Then as before, $x \mapsto V_{z}(r)$ is $\kappa$-convex. Stability of $\kappa$-convexity under taking pointwise suprema, therefore, yields that

$$
V(x):=\sup _{z \in Z_{r}} V_{z}(x)
$$

is $\kappa$-convex in $x$. Moreover, obviously $Y=\{V \leq 0\}$ and, for every $\delta>0$ there exists $\varepsilon>0$ such that $|D V| \geq 1-\delta$ on $Y_{\varepsilon}^{0}=\{0<V \leq \varepsilon\}$.

Let $Y$ be a locally $\kappa$-convex subset of $X$, parametrized by a continuous function $V: X \rightarrow \mathbb{R}$ such that $Y=\{V \leq 0\}$. Define $Y_{r}^{0}:=\{0<V \leq r\}$. For $\delta>0$, choose $r>0$ such that on $\overline{Y_{r}^{0}}$ the function $V$ is $(\kappa-\delta)$-convex with $|D V| \geq 1-\delta$. Set $\kappa^{\prime}:=\kappa-\delta$.

For $x \in \overline{Y_{r}^{0}}$, let $T(x):=\min \left\{t \geq 0: x_{t} \in Y\right\}$, where $\left(x_{t}\right)_{t \geq 0}$ is the $\mathrm{EVI}_{\kappa^{\prime}}$-gradient flow for $V$ starting from $x_{0}=x$. Given $x, y \in \overline{Y_{r}^{0}}$, put $T(x, y):=\sup _{s \in[0,1]} \inf _{\gamma} T\left(\gamma_{s}\right)$ where the infimum is taken over geodesics $\left(\gamma_{s}\right)_{s \in[0,1]}$ connecting $x$ to $y$.

Lemma 2.13. Let $Y, V, \delta, r$ be as above. Then for all $x, y \in \overline{Y_{r}^{0}}$

$$
\frac{d\left(y_{T(y)}, x_{T(x)}\right)}{d(y, x)} \leq e^{-(\kappa-\delta) T(x, y)} .
$$

Proof. Let us first consider the case that $\left(x_{t}\right)_{0 \leq t \leq T(x)}$ and $\left(y_{t}\right)_{0 \leq t \leq T(y)}$ as well as all geodesics connecting $x_{t}$ and $y_{t}$ for $t \leq \tau:=T(x) \wedge T(y)$ lie in one chart $X_{i}$. Set $\sigma:=|T(x)-T(y)|$. Then for $0 \leq t \leq \tau$ we have, by (6) , that $d\left(y_{t}, x_{t}\right) \leq e^{-\kappa^{\prime} t} d(y, x)$. In particular,

$$
\frac{d\left(y_{\tau}, x_{\tau}\right)}{d(y, x)} \leq e^{-\kappa^{\prime} \tau}
$$


Assume first that $0 \leq T(x) \leq T(y)$. Then $\tau=T(x)$ and $\sigma+\tau=T(y)$. Consider (5) with observation point $z=x_{\tau}$ and $\mathrm{EVI}_{\kappa^{\prime}}$-gradient flow $\left(y_{\tau+t}\right)_{t \geq 0}$ starting in $y_{\tau}$. Then, due to (5),

$$
\frac{1}{2} \frac{\partial}{\partial t} d^{2}\left(y_{\tau+t}, x_{\tau}\right) \leq-\frac{\kappa^{\prime}}{2} d^{2}\left(y_{\tau+t}, x_{\tau}\right)+V\left(x_{\tau}\right)-V\left(y_{\tau+t}\right) .
$$

Since $V\left(x_{\tau}\right)=V\left(x_{T(x)}\right)=0 \leq V\left(y_{\tau+t}\right)$ for $t \in[0, \sigma]$,

$$
\frac{\partial}{\partial t} d\left(y_{\tau+t}, x_{\tau}\right) \leq-\frac{\kappa^{\prime}}{2} d\left(y_{\tau+t}, x_{\tau}\right) .
$$

By Gronwall's lemma,

$$
d\left(y_{\tau+t}, x_{\tau}\right) \leq e^{-\frac{\kappa^{\prime}}{2} t} d\left(y_{\tau}, x_{\tau}\right), \quad \forall t \in[0, \sigma] .
$$

Setting $t=\sigma$ yields

$$
d\left(y_{T(y)}, x_{T(x)}\right) \leq e^{-\frac{\kappa^{\prime}}{2} \sigma} d\left(y_{\tau}, x_{\tau}\right) .
$$

Interchanging the roles of $x$ and $y$ in the above paragraph, we obtain the same estimate (9) also in the case $T(x) \geq T(y) \geq 0$. Combining (9) and (8), we obtain

$$
\frac{d\left(y_{T(y)}, x_{T(x)}\right)}{d(y, x)} \leq e^{-\frac{\kappa^{\prime}}{2}(\sigma+2 \tau)} \leq e^{-\frac{\kappa^{\prime}}{2}(T(x)+T(y))} .
$$

Now let us consider the general case. Given starting points $x, y \in \overline{Y_{r}^{0}}$, choose points $\gamma^{r_{k}}$ for $k=0,1, \ldots, n$ on the connecting geodesic with sufficiently small distance between consecutive points and apply the previous argument to the flows starting in pairs of points $\gamma^{r_{k}}$ and $\gamma^{r_{k+1}}$. It finally yields

$$
\begin{aligned}
d(x, y) & =\sum_{k} d\left(\gamma^{r_{k}}, \gamma^{r_{k+1}}\right) \\
& \geq \sum_{k} e^{\frac{\kappa^{\prime}}{2}\left(T\left(\gamma^{r_{k}}\right)+T\left(\gamma^{r_{k+1}}\right)\right)} d\left(\gamma_{T\left(\gamma^{r_{k}}\right)}^{r_{k}}, \gamma_{T\left(\gamma^{r_{k+1}}\right)}^{r_{k+1}}\right) \\
& \geq e^{\kappa^{\prime} T(x, y)} d\left(x_{T(x)}, y_{T(y)}\right) .
\end{aligned}
$$

\subsection{The convexity transform}

Throughout the sequel, let $Y=\{V \leq 0\}$ be a locally $\kappa$-convex subset of $X$ for some $\kappa \leq 0$.

Definition 2.14. (i) For a function $\phi: Y \rightarrow(0, \infty)$ that is bounded and bounded away from zero, define a metric $\phi \odot d$ on $Y$ by

$$
(\phi \odot d)(x, y):=\inf \left\{\int_{0}^{1} \phi\left(\gamma_{t}\right)\left|\dot{\gamma}_{t}\right| d t: \quad \gamma:[0,1] \rightarrow X \text { abs. continuous, } \gamma_{0}=x, \gamma_{1}=y\right\}
$$

(ii) For every $\kappa^{\prime} \leq \kappa$, put $d_{\kappa^{\prime}}:=\phi_{\kappa^{\prime}} \odot d$ with $\phi_{\kappa^{\prime}}(x):=e^{-\kappa^{\prime} V(x)}$.

Note that $-\kappa^{\prime} \geq 0$, thus $-\kappa^{\prime} V \geq 0$ on $X \backslash Y$ whereas $-\kappa^{\prime} V \leq 0$ on $Y$.

Put $Y_{r}:=\{z \in X: V(z) \leq r\}$. The following result is a consequence of Lemma 2.13,

Corollary 2.15. For any $\kappa^{\prime}<\kappa$, there exists $r>0$ such that for all $x \in Y_{r}$

$$
\limsup _{y \rightarrow x} \frac{d_{\kappa^{\prime}}\left(y_{T(y)}, x_{T(x)}\right)}{d_{\kappa^{\prime}}(y, x)} \leq 1,
$$

with strict inequality if $x \in Y_{r} \backslash Y$. 
Proof. Given $\kappa^{\prime}<\kappa$ there exist $\delta>0$ such that $\kappa^{\prime}<(1-\delta)^{-2} \cdot(\kappa-\delta)$. By assumption, there exist $r>0$ such that $V$ is $(\kappa-\delta)$-convex and $|D V|^{2} \geq(1-\delta)$ on $Y_{r} \backslash Y$. Since $\left(x_{t}\right)$ is a gradient flow in the sense of Definition 1.3. it is easy to see that $\frac{\partial}{\partial t} V\left(x_{t}\right)=-\left|D V\left(x_{t}\right)\right|^{2}$ for every $t>0$. Therefore, $V\left(x_{t}\right)<-(1-\delta)^{2} t+V(x)$ as long as $x_{t}$ does not leave $Y_{r} \backslash Y$. Thus $T(x)(1-\delta)^{2}<V(x)$ for all $x \in Y_{r} \backslash Y$ and, of course, $T(x)=0$ for $x \in Y$. Hence, by Lemma 2.13 (more precisely, by (10) ) for all $x, y \in Y_{r}$ sufficiently close to each other (such that they lie in a common set $X_{i}$ )

$$
\frac{d\left(y_{T(y)}, x_{T(x)}\right)}{d(y, x)} \leq e^{-\frac{\kappa-\delta}{2}(T(x)+T(y))} \leq e^{-\frac{\kappa^{\prime}}{2}(V(x)+V(y))}
$$

and, moreover, $\frac{d\left(y_{T(y)}, x_{T(x)}\right)}{d(y, x)}<e^{-\frac{\kappa^{\prime}}{2}(V(x)+V(y))}$ if $x \notin Y$. Noting that $\phi_{\kappa^{\prime}}\left(x_{T(x)}\right)=1$, we obtain for all $x \in Y_{r}$

$$
\begin{aligned}
\limsup _{y \rightarrow x} \frac{d_{\kappa^{\prime}}\left(y_{T(y)}, x_{T(x)}\right)}{d_{\kappa^{\prime}}(y, x)} & \leq \limsup _{y \rightarrow x} \frac{d_{\kappa^{\prime}}\left(y_{T(y)}, x_{T(x)}\right)}{d\left(y_{T(y)}, x_{T(x)}\right)} \frac{d\left(y_{T(y)}, x_{T(x)}\right)}{d(y, x)} \frac{d(y, x)}{d_{\kappa^{\prime}}(y, x)} \\
& \leq \phi_{\kappa^{\prime}}\left(x_{T(x)}\right) e^{-\kappa^{\prime} V(x)} \frac{1}{\phi_{\kappa^{\prime}}(x)}=1
\end{aligned}
$$

with strict inequality in the last line if $x \notin Y$.

Corollary 2.16. For $\kappa^{\prime}<\kappa$ and $r>0$ as in Corollary 2.15, consider the map

$$
\Phi: Y_{r} \rightarrow Y, \quad x \mapsto x_{T(x)} .
$$

For any Lipschitz curve $\left(\gamma_{s}\right)_{0 \leq s \leq 1}$ in $Y_{r}$ define a Lipschitz curve $\left(\tilde{\gamma}_{s}\right)_{0 \leq s \leq 1}$ in $Y$ by $\tilde{\gamma}_{s}:=\Phi\left(\gamma_{s}\right)$. Then

$$
\text { length }_{d_{\kappa^{\prime}}}(\tilde{\gamma}) \leq \operatorname{length}_{d_{\kappa^{\prime}}}(\gamma)
$$

with strict inequality if the original curve $\left(\gamma_{s}\right)_{0 \leq s \leq 1}$ does not completely lie in $Y$ (or, in other words, if $\tilde{\gamma} \neq \gamma)$.

Theorem 2.17 ('Convexification Theorem'). $Y$ is locally geodesically convex in $\left(X, d_{\kappa^{\prime}}\right)$ for any $\kappa^{\prime}<\kappa$.

Proof. With $\kappa^{\prime}<\kappa$ and $r>0$ as in Corollary 2.15 and $d^{\prime}:=d_{\kappa^{\prime}}$, choose a countable family of open sets $U_{i} \subset X$ with $d^{\prime}$-diameter $2 \delta_{i}$ such that

$$
Y \subset \bigcup_{i} U_{i}, \quad \bigcup_{i} B_{\delta_{i}}^{\prime}\left(U_{i}\right) \subset Y_{r}
$$

where $B_{\delta_{i}}^{\prime}\left(U_{i}\right)=\left\{x: d^{\prime}\left(x, U_{i}\right)<\delta_{i}\right\}$.

- Then every $d^{\prime}$-geodesic $\left(\gamma_{s}\right)_{0 \leq s \leq 1}$ in $X$ with endpoints in one of the sets $Y \cap U_{i}$ will completely lie in $Y_{r}$.

- According to Corollary 2.16, the fact that $\left(\gamma_{s}\right)_{0 \leq s \leq 1}$ is a $d^{\prime}$-geodesic with endpoints in $Y$ implies that it has to lie completely in $Y$. Otherwise, the map $\Phi$ would map it onto a shorter curve with the same endpoints.

\section{Controlling the curvature}

Our next goal is to prove that the convexification transform introduced in the previous section preserves generalized lower Ricci curvature bounds. More precisely, we will show that $\left(X, d^{\prime}, m\right)$ satisfies an $\operatorname{RCD}\left(K^{\prime}, \infty\right)$-condition provided that $(X, d, m)$ satisfies an $\operatorname{RCD}^{*}(K, N)$-condition. Recall that the metric measure space $(X, d, m)$ is said to be a $\operatorname{RCD}^{*}(K, N)$ space if the reduced curvature dimension condition $\mathrm{CD}^{*}(K, N)$ (defined in [BS10, Definition 2.3]) is satisfied and the Cheeger energy is a quadratic form on $L^{2}(X, m)$.

Another goal is to prove that the $\operatorname{CD}\left(K^{\prime}, \infty\right)$-condition is preserved if we replace the metric measure space by a locally geodesically convex subset. 


\subsection{Curvature control for convexity transform}

Proposition 3.1 (EKS15, Proposition 3.3]). Let $(X, d, m)$ satisfy $\operatorname{RCD}^{*}(K, N)$ with $N<$ $\infty$ and let $V: X \rightarrow(-\infty, \infty)$ be a lower bounded, $\kappa$-convex function with $|D V|^{2} \leq C_{1}$. Then for each $N^{\prime} \in(N, \infty]$ the drift-transformed metric measure space $\left(X, d, e^{-V} m\right)$ satisfies $\operatorname{RCD}^{*}\left(K^{\prime}, N^{\prime}\right)$ with $K^{\prime}:=K+\kappa+\frac{C_{1}}{N^{\prime}-N}$.

Proposition 3.2. Let $(X, d, m)$ be an $\operatorname{RCD}^{*}\left(K^{\prime}, N^{\prime}\right)$ space with finite $K^{\prime}, N^{\prime} \in \mathbb{R}, N^{\prime} \geq 1$. Then for every $w \in \operatorname{TestF}(X)$ the conformally transformed metric measure space $\left(X, e^{w} \odot d, e^{N^{\prime} w} m\right)$ satisfies $\mathrm{RCD}^{*}\left(K^{\prime \prime}, N^{\prime}\right)$ for each $K^{\prime \prime} \in \mathbb{R}$ such that

$$
e^{2 w} K^{\prime \prime} \leq K^{\prime}-\Delta w-\left(N^{\prime}-2\right) \Gamma(w)-\frac{N^{\prime}-2}{\Gamma(f)}\left(\operatorname{Hess}_{w}(\nabla f, \nabla f)-\Gamma(w, f)^{2}\right)
$$

a.e. on $X$ for each $f \in \operatorname{Test} F(X)$.

Proof. This is proved in [HM, Corollary 3.15] under the hypothesis that an exponential volume growth condition holds. The volume growth condition, however, follows from $\operatorname{CD}(K, \infty)$, by [Stu06, Theorem 4.24].

Corollary 3.3. Let $(X, d, m)$ be an $\operatorname{RCD}^{*}(K, N)$ space with $N<\infty$ and let $w \in \operatorname{TestF}(X)$ be a $\kappa$-convex function. Then the transformed metric measure space $\left(X, e^{w} \odot d, m\right)$ satisfies $\operatorname{RCD}^{*}\left(K^{\prime \prime}, N^{\prime}\right)$ provided that $N^{\prime} \in(N, \infty)$ and

$$
e^{2 w} K^{\prime \prime} \leq K^{\prime}-\Delta w+2 \Gamma(w)-\frac{N^{\prime}-2}{\Gamma(f)}\left(\operatorname{Hess}_{w}(\nabla f, \nabla f)-\Gamma(w, f)^{2}\right)
$$

a.e. on $X$ for each $f \in \operatorname{Test} F(X)$, where $K^{\prime}:=K+N^{\prime} \kappa+N^{\prime 2} \frac{C_{1}}{N^{\prime}-N}$.

Proof. For given $N^{\prime}>N$, apply first Proposition 3.1 with $V:=N^{\prime} w$. Then apply Proposition 3.2 with $e^{-N^{\prime} w} m$ in the place of $m$ and thus with $\Delta-N^{\prime} \Gamma(w,$.$) in the place of \Delta$.

We are now going to apply these results to the convexification transform as introduced in the previous section. To do so, we have to slightly enforce the assumptions on the functions $V$ used for defining $\kappa$-regular sets.

Definition 3.4. A subset $Y \subset X$ is called regularly $\kappa$-convex if for every $\varepsilon \in(0,1]$ there exists a $\kappa$-convex map $V=V_{\varepsilon}: X \rightarrow(-\varepsilon, \infty)$ such that

$$
Y=\{x \in X: V(x) \leq 0\} \quad \text { and } \quad \lim _{r \rightarrow 0} \inf _{\{x \in X: 0<V(x) \leq r\}}|D V|(x) \geq 1
$$

and in addition

(i) $V \in \operatorname{TestF}(X)$,

(ii) $\Delta V \leq C_{2}$ for some constant $C_{2} \in \mathbb{R}$,

(iii) $\operatorname{Hess}_{V}(\nabla f, \nabla f) \leq C_{3} \Gamma(f)$ for all $f \in \operatorname{TestF}(X)$.

Functions $V$ with these properties will be called regularly $\kappa$-convex.

Remark 3.5. In the Riemannian setting, we can simply start with the function $V_{\epsilon}$ for $\epsilon=1$ and construct all other $V_{\epsilon}$ by truncating $V_{1}$ at level $-\epsilon$ and smoothing the resulting function such that it still matches the requested bounds on the second derivatives. In general, however, such a smoothing might not exist. Note that (iii) implies (ii) if, as in the Riemannian setting, $\Delta V=\operatorname{tr} \operatorname{Hess}_{V}$. 
Theorem 3.6. Let $(X, d, m)$ be an $\operatorname{RCD}^{*}(K, N)$-space with $N<\infty$ and let $V: X \rightarrow \mathbb{R}$ be a regularly $\kappa$-convex function for some $\kappa \leq 0$. Then for every $\kappa^{\prime}<\kappa$ the $m m$-space $\left(X, e^{-\kappa^{\prime} V} \odot\right.$ $d, m)$ satisfies $\operatorname{RCD}^{*}\left(K^{\prime \prime}, N^{\prime}\right)$ with $N^{\prime}=N+1$ and

$$
\left.K^{\prime \prime}=e^{2 \kappa^{\prime} C_{0}}\left[K-(N+1) \kappa \kappa^{\prime}+(N+1)^{2} \kappa^{\prime 2} C_{1}+\kappa^{\prime} C_{2}-(N-3) \kappa^{\prime 2} C_{1}+(N-1) \kappa^{\prime} C_{3}\right)\right],
$$

where $C_{i}$ for $i=0, \ldots, 4$ are finite constants with $V \leq C_{0}, \Gamma(V) \leq C_{1}$ and $C_{2}, C_{3}$ as in Definition 3.4.

Proof. Let $w:=-\kappa^{\prime} V$ and $N^{\prime}=N+1$. Then $w \in \operatorname{TestF}(X)$ and $N^{\prime} w=-\kappa^{\prime} N^{\prime} V$ is $\left(-\kappa \kappa^{\prime} N^{\prime}\right)$ convex with $\left|D\left(N^{\prime} w\right)\right|^{2} \leq \kappa^{\prime 2} N^{\prime 2} C_{1}$. By Corollary 3.3, $\left(X, e^{-\kappa^{\prime} V} \odot d, m\right)$ satisfies $\mathrm{RCD}^{*}\left(K^{\prime \prime}, N^{\prime}\right)$ provided that for each $f \in \operatorname{TestF}(\mathrm{X})$,

$$
K^{\prime \prime} \leq e^{-2 w}\left[K^{\prime}-\Delta w+2 \Gamma(w)-\frac{N^{\prime}-2}{\Gamma(f)}\left(\operatorname{Hess}_{w}(\nabla f, \nabla f)-\Gamma(w, f)^{2}\right)\right] .
$$

The right hand side is obviously bounded from below by

$$
\begin{aligned}
& e^{2 \kappa^{\prime} V}\left[K^{\prime}+\kappa^{\prime} \Delta V+2 \kappa^{\prime 2} \Gamma(V)-\frac{N^{\prime}-2}{\Gamma(f)}\left(\operatorname{Hess}_{-\kappa^{\prime} V}(D f, D f)-\Gamma\left(-\kappa^{\prime} V, f\right)^{2}\right)\right] \\
& \geq e^{2 \kappa^{\prime} V}\left[K^{\prime}-\left(-\kappa^{\prime}\right) \Delta V+2 \kappa^{\prime 2} \Gamma(V)+\frac{N^{\prime}-2}{\Gamma(f)}\left(\kappa^{\prime} \operatorname{Hess}_{V}(\nabla f, \nabla f)+\kappa^{\prime 2} \Gamma(V, f)^{2}\right)\right] \\
& \geq e^{2 \kappa^{\prime} V}\left[K^{\prime}-\left(-\kappa^{\prime}\right) \Delta V+2 \kappa^{\prime 2} \Gamma(V)+(N-1)\left(\kappa^{\prime} C_{3}-\kappa^{\prime 2} \Gamma(V)\right)\right] \\
& \left.=e^{2 \kappa^{\prime} C_{0}}\left[K^{\prime}+\kappa^{\prime} C_{2}-(N-3) \kappa^{\prime 2} C_{1}+(N-1) \kappa^{\prime} C_{3}\right)\right]
\end{aligned}
$$

where $K^{\prime}:=K-N^{\prime} \kappa \kappa^{\prime}+N^{\prime 2} \kappa^{\prime 2} C_{1}$.

\subsection{Curvature control for restriction to locally geodesically convex sets}

Theorem 3.7. Let $(X, d, m)$ be a metric measure space that satisfies $C D(K, \infty)$ for some $K \in \mathbb{R}$. Let $Y \subset X$ be a closed subset that is locally geodesically convex and satisfies $m(Y)>0, Y=\overline{Y^{o}}$ and $d_{Y}<\infty$ on $Y \times Y$. Then $\left(Y, d_{Y}, m_{Y}\right)$ also satisfies $C D(K, \infty)$. Here $d_{Y}$ denotes the induced length metric on $Y$, i.e.

$$
d_{Y}(x, y):=\inf \left\{\int_{0}^{1}\left|\dot{\gamma}_{t}\right| d t: \quad \gamma:[0,1] \rightarrow Y \text { abs. continuous, } \gamma_{0}=x, \gamma_{1}=y\right\}
$$

and $m_{Y}$ denotes the restriction of $m$ to $Y$.

More generally, if $(X, d, m)$ satisfies $C D^{*}(K, N)$ or $R C D^{*}(K, N)$ then the same is true for $\left(Y, d_{Y}, m_{Y}\right)$.

The condition $Y=\overline{Y^{o}}$ guarantees that $m_{Y}$ has full topological support. The conditions $m(Y)>0$ and $d_{Y}<\infty$ avoid pathologies. Under $Y=\overline{Y^{o}}$, we have $d_{Y}<\infty$ if and only if any two points $x, y \in Y$ can be connected by a rectifiable curve which completely lies in $Y$.

Proof. We first show that $\left(Y, d_{Y}\right)$ is locally compact. If $Y$ is locally geodesically convex with open covering $\bigcup_{i} X_{i}$, then $d(x, y)=d_{Y}(x, y)$ whenever $x, y \in X_{i}$ for some $i$. Let $y \in Y \cap X_{i}$ for some $X_{i}$. By the local compactness of $(X, d)$, there exists a neighborhood $U \subset X$ of $y$ that is compact in $(X, d)$. Let $r:=d\left(y, \overline{X_{i}} \backslash X_{i}\right)$ and set $V:=U \cap Y \cap B(y, r / 2)$. Then $V$ is a neighborhood of $y$ and since $d(x, z)=d_{Y}(x, z)$ for any $x, z \in V$ it follows that $V$ is compact.

We note that $\left(Y, d_{Y}\right)$ is a proper metric space since it is locally compact and geodesic. For any pair of probability measures $\mu_{0}, \mu_{1}$ supported in some $Y \cap X_{i}$, the $d$-Wasserstein geodesic from $\mu_{0}$ to $\mu_{1}$ coincides with a $d_{Y}$-Wasserstein geodesic. In particular, there exists a $d_{Y}$-Wasserstein geodesic along which the entropy is $K$-convex. Thus $\left(Y, d_{Y}, m_{Y}\right)$ locally has curvature $\geq K$ in 
the sense of [Stu06, Definition 4.5(iii)]. The Local-to-Global-Theorem [Stu06, Theorem 4.17], then implies that it has curvature $\geq K^{\prime}$ for each $K^{\prime}<K$. Local compactness allows to conclude that is has curvature $\geq K$ or, in other words, that it satisfies $\operatorname{CD}(K, \infty)$. Indeed, it suffices to verify the $K$-convexity of the entropy along optimal transports between pairs of probability measures with bounded supports [Vil09, Corollary 29.23]. These transports will stay within bounded, hence compact, sets.

\section{Heat flow on $Y$ as gradient flow for the entropy}

\subsection{Heat flow on $X$}

In this section, we assume that the (complete, locally compact, geodesic) metric measure space $(X, d, m)$ has full topological support and satisfies

$$
\int e^{-C d^{2}(x, z)} d m(x)<\infty
$$

for some $C \in \mathbb{R}, z \in X$. Note that (11) follows from the $\operatorname{CD}(K, \infty)$ condition, see Stu06, Theorem 4.24].

Definition 4.1. A function $G \in L^{2}(X, m)$ is a relaxed gradient of $f \in L^{2}(X, m)$ if there exists a sequence of Lipschitz (with respect to $d$ ) functions $f_{n} \in L^{2}(X, m)$ such that $f_{n} \rightarrow f$ in $L^{2}(X, m)$, $\left|D f_{n}\right|$ converges weakly to some $\tilde{G} \in L^{2}(X, m)$ and $\tilde{G} \leq G m$-almost everywhere in $X$. The minimal relaxed gradient $|D f|_{*}$ is the relaxed gradient of $f$ which has minimal $L^{2}$-norm among all relaxed gradients of $f$.

Suppose (11) holds. Then, by [AGS14a, Theorem 6.2], the minimal relaxed gradient $|D f|_{*}$ coincides with the $\mathcal{T}$-minimal weak upper gradient defined in AGS14a, Definition 5.11] with respect to the collection $\mathcal{T}$ of all test plans concentrated on the absolutely continuous curves in $X$ with bounded compression, with the upper gradient from [Che99, HK98, as well as with the Newtonian gradient from Sha00. The minimal relaxed gradient $|D f|_{*}$ is also the same as the weak gradient in [HM], by [AGS14a, Lemma 4.3, Lemma 4.4].

Let $\operatorname{Lip}_{c}(X)$ is the space of Lipschitz continuous (with respect to $d$ ) functions with compact support in $X$.

Lemma 4.2. (i) The Cheeger energy $\mathrm{Ch}(f): L^{2}(X, m) \rightarrow \mathbb{R}$ as defined in (1) coincides with

$$
\operatorname{Ch}(f)=\frac{1}{2} \int_{X}|D f|_{*}^{2} d m,
$$

when $f$ has a minimal relaxed gradient, and $\mathrm{Ch}(f):=+\infty$ otherwise.

(ii) The space of Lipschitz functions $f$ with $|D f| \in L^{2}$ is dense in the domain Dom(Ch):= $\left\{f \in L^{2}(X, m): \operatorname{Ch}(f)<+\infty\right\}$ for the norm $\|f\|_{\mathcal{F}}:=\left(\operatorname{Ch}(f)+\int_{X} f^{2} d m\right)^{1 / 2}$. The domain Dom $(\mathrm{Ch})$ of the Cheeger energy, also denoted by $\mathcal{F}$ or $W^{1,2}(X, d, m)$, is complete and dense in $L^{2}(X, m)$.

(iii) The Cheeger energy is strongly local, Markovian, and regular with core Lip $p_{c}(X)$.

Proof. (i) This is immediate from [AGS14a, Lemma 4.3(c)].

(ii) From (i) and (1), we see that the space of Lipschitz continuous functions $f$ with $|D f| \in$ $L^{2}(X, m)$ is dense in $\operatorname{Dom}(C h)$. The density of $\operatorname{Dom}(\mathrm{Ch})$ in $L^{2}(X, m)$ is proved in AGS14a, Theorem 4.5]. Completeness follows from either [AGS14a, Remark 4.6] or [Che99, Theorem $2.7]$.

(iii) The strong locality and Markovian property follow from [AGS14a, Proposition 4.8]. Regularity follows from (ii) and the fact that we can use the distance function to construct cutoff functions that are Lipschitz continuous with slope 1. 
Lemma 4.3. (i) For each $f_{0} \in L^{2}(X, m)$ there exists a unique gradient flow $\left(f_{t}\right)_{t \in[0, \infty)} \subset$ $L^{2}(X, m)$ for the Cheeger energy $C h$ which starts in $f_{0}$. It is called the heat flow starting in $f_{0}$.

(ii) If $(X, d, m)$ satisfies $C D(K, \infty)$ then for each $f_{0} \in L^{2}(X, m)$ with $f_{0} m \in \mathcal{P}_{2}(X)$ the following are equivalent:

- $t \mapsto f_{t}$ is a gradient flow for $\mathrm{Ch}$ in $L^{2}(X, m)$

- $t \mapsto \mu_{t}=f_{t} m$ is a gradient flow for $\operatorname{Ent}_{m}$ in $\left(\mathcal{P}_{2}(X), W_{2}\right)$.

Proof. (i) This follows from existence theory of gradient flows on Hilbert spaces, e.g. AGS05, Corollary 2.4.11], together with the convexity of the Cheeger energy [AGS14a, Theorem 4.5].

(ii) is proved in AGS14a, Theorem 9.3(iii)].

Suppose now that $(X, d, m)$ satisfies $\operatorname{RCD}(K, \infty)$. By polarization of $2 \mathrm{Ch}$, we obtain a strongly local symmetric Dirichlet form

$$
\mathcal{E}(f, f):=2 \operatorname{Ch}(f), \quad f \in \operatorname{Dom}(\mathcal{E}):=\operatorname{Dom}(\mathrm{Ch}) .
$$

Its infinitesimal generator is the Laplacian on $X$, defined as the unique non-positive definite self-adjoint operator $(\Delta, \operatorname{Dom}(\Delta))$ on $L^{2}(X, m)$ with $\operatorname{Dom}(\Delta) \subset \mathcal{F}$ and

$$
\mathcal{E}(u, v)=-\int \Delta u v d m \quad \forall u \in \operatorname{Dom}(\Delta), v \in \mathcal{F} .
$$

$(\mathcal{E}, \mathcal{F})$ is regular with core $\operatorname{Lip}_{\mathrm{c}}(X)$, and admits a carré du champ which we denote by $\Gamma(\cdot, \cdot)$. In particular,

$$
\Gamma(f, f)=|D f|_{*}^{2}=|\nabla f|^{2}, \quad \forall f \in \mathcal{F} .
$$

Here, the gradient $\nabla f$ is defined as an element of the tangent module $L^{2}(T X)$, see Gig. Moreover, the metric $d$ is the length-metric induced by $(\mathcal{E}, \mathcal{F})$. We recall that the $\Gamma_{2}$-operator is defined as

$$
\Gamma_{2}(f, g):=\Gamma(f, \Delta g)-\frac{1}{2} \Delta \Gamma(f, g) .
$$

The Hessian is defined as

$$
\operatorname{Hess}_{w}(\nabla f, \nabla f)=\Gamma(f, \Gamma(w, f))-\frac{1}{2} \Gamma(w, \Gamma(f, f)),
$$

and $\operatorname{Hess}_{w}$ may also be denoted as Hess $w$.

\subsection{Neumann heat flow on $Y \subset X$}

Let $(X, d, m)$ satisfy (11). Let $Y \subset X$ be an arbitrary closed subset with $m(Y)>0$. Let $Y^{o}$ be the interior of $Y$. We obtain a new metric measure space $\left(Y, d_{Y}, m_{Y}\right)$ with $m_{Y}:=\left.m\right|_{Y}$ and $d_{Y}$ being the induced length metric on $Y$. We will assume $d_{Y}<\infty$ on $Y \times Y$. (Alternatively, one could study the heat flow on each connected component of $Y$.) This new metric measure space again satisfies (11) (since $d_{Y} \geq d$ ).

For $f: Y \rightarrow \mathbb{R}$ put

$$
\left|D_{Y} f\right|(x):=\limsup _{y \rightarrow x, y \neq x} \frac{|f(y)-f(x)|}{d_{Y}(y, x)}, \quad x \in Y .
$$

Analogous to Definition 4.1 we let $\left|D_{Y} f\right|_{*}$ denote the minimal relaxed gradient of $f \in L^{2}\left(Y, m_{Y}\right)$ defined in terms of approximation by Lipschitz functions on $\left(Y, d_{Y}\right)$. 
Lemma 4.4. Under the standing assumptions of this subsection, the following holds.

(i) For each $f: X \rightarrow \mathbb{R}$ let $\tilde{f}=\left.f\right|_{Y}$ denote its restriction to $Y$. Then

$$
\left|D_{Y} \tilde{f}\right| \leq|D f| \quad \text { on } Y \quad \text { and } \quad\left|D_{Y} \tilde{f}\right|=|D f| \quad \text { on } Y^{o} .
$$

(ii) For each $f: X \rightarrow \mathbb{R}$ with compact support in $Y^{o}$

$$
\left|D_{Y} f\right|_{*}=|D f|_{*} \quad m \text {-a.e. on } X \text {. }
$$

Here and in the sequel $\left|D_{Y} f\right|_{*}$ will be extended to all of $X$ with value 0 outside of $Y$.

Proof. (i) For each $x \in Y^{o}$ there exists $r>0$ such that $B_{2 r}(x) \subset Y$. Thus $d_{Y}(y, x)=d(y, x)$ for all $y \in B_{r}(x)$ and the assertion follows from the definitions of $D_{Y}$ and $D$.

(ii) Let $f$ be given with compact support $Z \subset Y^{o}$.

To prove the $\leq$-assertion, let $g_{n} \in \operatorname{Lip}(X, d)$ be given with $g_{n} \rightarrow f$ in $L^{2}(X, m)$ and $\left|D g_{n}\right| \rightarrow$ $|D f|_{*}$ weakly in $L^{2}(X, m)$. Denote by $\tilde{f}_{n}$ and $\tilde{g}$ the restrictions of $f_{n}$ and $g$, resp., to the set $Y$. Then obviously $\tilde{g}_{n} \in \operatorname{Lip}\left(Y, d_{Y}\right)$ (recall that $\left.\left|D_{Y} \tilde{g}_{n}\right| \leq\left|D g_{n}\right|\right)$ and $\tilde{g}_{n} \rightarrow \tilde{f}$ in $L^{2}\left(Y, m_{Y}\right)$. Moreover, as $n \rightarrow \infty$

$$
\left|D_{Y} \tilde{g}_{n}\right| \leq\left|D g_{n}\right| \rightarrow|D f|_{*}
$$

weakly in $L^{2}\left(Y, m_{Y}\right)$. Thus $|D f|_{*}$ is a relaxed upper $d_{Y}$-gradient for $\tilde{f}$ which yields the claim: $\left|D_{Y} \tilde{f}\right|_{*} \leq|D f|_{*}$ a.e. on $X$.

To prove the converse, let $g_{n} \in \operatorname{Lip}\left(Y, d_{Y}\right)$ be given with $g_{n} \rightarrow f$ in $L^{2}\left(Y, m_{Y}\right)$ and $\left|D g_{n}\right| \rightarrow$ $\left|D_{Y} f\right|_{*}$ weakly in $L^{2}\left(Y, m_{Y}\right)$. Let $Z$ be the compact support of $f$. Choose $r>0$ with $B_{r}(Z) \subset$ $Y^{o}$ and let $\chi: X \rightarrow \mathbb{R}$ denote the $d$-Lipschitz ('cut-off') function with $\chi=1$ on $Z, \chi=0$ outside of $B_{r}(Z)$, and $\left|\chi^{\prime}\right| \leq 1 / r$ on $X$. Extend $g_{n}$ and $f$ to all of $X$ with value 0 outside of $Y$ and define $\hat{g}_{n}: X \rightarrow \mathbb{R}$ by $g_{n}=\chi \cdot g_{n}$. Then $\hat{g}_{n} \in \operatorname{Lip}(X, d)$ and $\hat{g}_{n} \rightarrow f$ in $L^{2}(X, m)$. Moreover, as $n \rightarrow \infty$ weakly in $L^{2}(X, m)$

$$
\left|D \hat{g}_{n}\right| \leq \chi \cdot\left|D g_{n}\right|+\frac{1}{r} \cdot 1_{Y \backslash Z} \cdot g_{n} \rightarrow \chi \cdot\left|D_{Y} f\right|_{*}=\left|D_{Y} f\right|_{*}
$$

since $1_{Y \backslash Z} \cdot g_{n} \rightarrow 0$ in $L^{2}(X, m)$ and since $\left|D_{Y} f\right|_{*}=0$ a.e. outside of the compact support $Z$ of $f$. Thus $\left|D_{Y} f\right|_{*}$ is a relaxed upper $d$-gradient for $f$ on $X$. Therefore, $|D \tilde{f}|_{*} \leq\left|D_{Y} f\right|_{*}$ a.e. on $X$.

Let $\mathrm{Ch}_{Y}: L^{2}\left(Y, m_{Y}\right) \rightarrow[0, \infty]$ denote the Cheeger energy for the metric measure space $\left(Y, d_{Y}, m_{Y}\right)$. From Lemma 4.3(i), we obain the existence of the gradient flow for the Cheeger energy on $Y$.

Proposition 4.5. Under the standing assumptions of this subsection, for each $f_{0} \in L^{2}\left(Y, m_{Y}\right)$ there exists a unique gradient flow $\left(f_{t}\right)_{t \in[0, \infty)} \subset L^{2}\left(Y, m_{Y}\right)$ for the Cheeger energy $\mathrm{Ch}_{Y}$ which starts in $f_{0}$. It is called the heat flow on $Y$ starting in $f_{0}$.

To distinguish this heat flow on $Y$ from other 'heat flows' (with different 'boundary conditions') on might also call it 'Neumann heat flow on $Y$ '.

Let us analyze the Cheeger energy on $Y$ in more detail. In particular, we will identify it with the construction of the energy for the so-called 'reflected process' or 'Neumann Laplacian' as used in Dirichlet form theory and Markov process literature, e.g. [Sil74].

Recall the notation $\mathcal{F}=\operatorname{Dom}(\mathrm{Ch})$. For any open set $U \subset X$ let

$$
\mathcal{F}^{\text {loc }}(U):=\left\{f \in L_{\text {loc }}^{2}(U, m): \forall \text { open rel. compact } V \subset U, \exists g \in \mathcal{F},\left.f\right|_{V}=\left.g\right|_{V} m \text {-a.e. }\right\} \text {. }
$$

Analogously, we define $\mathcal{F}_{Y}$ and $\mathcal{F}_{Y}^{\text {loc }}(U)$ for $d_{Y}$-open sets $U \subset Y$. The minimal relaxed gradient $|D \cdot|_{*}$ can be extended to $\mathcal{F}^{\text {loc }}(U)$ by setting $|D f|_{*}:=|D g|_{*}$ on $V$ for $g \in \mathcal{F}$ with $f=g$ on $V$ ('locality property'). Similarly for $\left|D_{Y} \cdot\right|_{*}$. 
Lemma 4.6. Under the standing assumptions of this subsection, $\mathcal{F}_{Y}^{\text {loc }}\left(Y^{o}\right)=\mathcal{F}^{\text {loc }}\left(Y^{o}\right)$ and for each $f \in \mathcal{F}_{Y}^{\text {loc }}\left(Y^{o}\right)$

$$
|D f|_{*}=\left|D_{Y} f\right|_{*} \text { a.e. on } Y^{o} \text {. }
$$

Proof. By definition, a function $f$ lies in $\mathcal{F}^{\text {loc }}\left(Y^{o}\right)$ if and only if for each open $V$, relatively compact in $Y^{o}$ there exists $g \in \mathcal{F}$ with $f=g$ on $V$. By truncating $g$ outside of $V$, the latter is easily seen to be equivalent to the fact that for each open $V$, relatively compact in $Y^{o}$ there exists $g \in \mathcal{F}$ with compact support in $Y^{o}$ and with $f=g$ on $V$. By Lemma 4.4 this in turn is equivalent to the fact that for each open $V$, relatively compact in $Y^{o}$ there exists $g \in \mathcal{F}_{Y}$ with compact support in $Y^{o}$ and with $f=g$ on $V$. Following the previous argumentation, this finally is equivalent to $f \in \mathcal{F}_{Y}^{\text {loc }}\left(Y^{o}\right)$.

Moreover, for each such $V$ and $g$ with compact support in $Y^{\circ}$, Lemma 4.4 yields $|D g|_{*}=$ $\left|D_{Y} g\right|_{*}$ a.e. on $X$. Thus $|D f|_{*}=\left|D_{Y} f\right|_{*}$ a.e. on $V$. This proves the claim.

Theorem 4.7. Assume that $(X, d, m)$ satisfies (11) and that $Y$ is a closed subset of $X$ with $d_{Y}<\infty, m(Y)>0, m(\partial Y)=0$. Then

$$
\mathrm{Ch}_{Y}(f)=\int_{Y^{o}}|D f|_{*}^{2} d m
$$

for all $f \in \mathcal{F}^{\text {loc }}\left(Y^{o}\right)$ and

$$
\operatorname{Dom}\left(\mathrm{Ch}_{Y}\right)=\left\{f \in \mathcal{F}^{\mathrm{loc}}\left(Y^{o}\right): \int_{Y^{o}}\left[|D f|_{*}^{2}+f^{2}\right] d m<\infty\right\} .
$$

Proof. By definition, $\mathcal{F}_{Y}^{\text {loc }}\left(Y^{o}\right) \supset \mathcal{F}_{Y}$. The assumption $m(\partial Y)=0$ and Lemma 4.6 imply

$$
\mathrm{Ch}_{Y}(f)=\int_{Y}\left|D_{Y} f\right|_{*}^{2} d m=\int_{Y^{o}}\left|D_{Y} f\right|_{*}^{2} d m=\int_{Y^{o}}|D f|_{*}^{2} d m
$$

for all $f \in \mathcal{F}_{Y}^{\text {loc }}\left(Y^{o}\right)$. This proves the claim.

Corollary 4.8. Under the assumptions of Theorem 4.7: if $(X, d, m)$ is infinitesimally Hilbertian (that is, $\mathcal{F}$ is a Hilbert space for the inner product induced by the Cheeger energy) then so is $\left(Y, d_{Y}, m_{Y}\right)$.

We point out that the Cheeger energy on $Y$ is intrinsically defined on the metric measure space $\left(Y, d_{Y}, m_{Y}\right)$, while the right hand side of (13) is the restriction to $Y$ of the Cheeger energy on the ambient metric measure space $(X, d, m)$. Thus, Theorem 4.7justifies calling the gradient flow of $\mathrm{Ch}_{Y}$ the Neumann heat flow on $Y$. Finally, the following corollary relates $\mathrm{Ch}_{Y}$ to the Neumann Laplacian, the Neumann heat semigroup, and the reflected Brownian motion on $Y$. Each of these is defined either intrinsically on $Y$ or constructed from the ambient space by restriction and using Neumann boundary condition.

Corollary 4.9. Assume that $(X, d, m)$ is infinitesimally Hilbertian and satisfies (11) and that $Y$ is a closed subset of $X$ with $d_{Y}<\infty, m(Y)>0, m(\partial Y)=0$.

(i) Then $\mathrm{Ch}_{Y}$ is a quadratic form. By polarization it induces a Dirichlet form $\mathcal{E}_{Y}$ on $L^{2}\left(Y, m_{Y}\right)$ which is regular and strongly local with core $\operatorname{Lip}_{c}(Y)$. Its generator $\Delta^{Y}$, the Neumann Laplacian on $Y$, is a linear self-adjoint nonpositive operator.

(ii) The heat flow on $Y$, initially defined as the gradient flow for the Cheeger energy $\mathrm{Ch}_{Y}$ on $L^{2}\left(Y, m_{Y}\right)$, is given in terms of the linear heat semigroup $\left(P_{t}^{Y}\right)_{t>0}$ on $L^{2}$ generated by the Neumann Laplacian on $Y$.

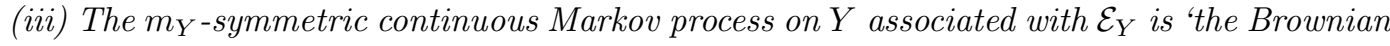
motion on $Y$ with reflecting boundary conditions'. 
Example 4.10 (Disc without ray). $U=\left\{(x, y) \in \mathbb{R}^{2}: x^{2}+y^{2}<1\right.$ and either $y \neq 0$ or $\left.x<0\right\}$ regarded as subset of $X=\mathbb{R}^{2}$, the latter being equipped with Euclidean distance $d$ and Lebesgue measure $m$. Put $Y=\bar{U}$. (Note that $Y^{o} \neq U$.) Then

$$
\operatorname{Dom}\left(\mathrm{Ch}_{Y}\right) \subsetneq \operatorname{Dom}\left(\mathrm{Ch}_{U}\right):=\left\{f \in \mathcal{F}^{l o c}(U): \int_{U}\left[|D f|_{*}^{2}+f^{2}\right] d m<\infty\right\} .
$$

For instance, the latter set contains the function $f$ with $f(x, y)=+x$ on the upper right quadrant, $f(x, y)=-x$ on the lower right quadrant and $f(x, y)=0$ on the left half space. Due to this discontinuity and lack of differentiability, $f$ is clearly not in $\operatorname{Dom}\left(\mathrm{Ch}_{Y}\right)$.

Example 4.11 (Plane without cusp). Let $\Omega=\left\{(x, y) \in \mathbb{R}^{2}: 0<x<1,0<y<x^{\alpha}\right\}$ where $\alpha>1$. Let $Y=\mathbb{R}^{2} \backslash \Omega$. According to [Maz11, Example 3 in Section 1.5.1], Sobolev functions on $Y^{o}=\mathbb{R}^{2} \backslash \bar{\Omega}$ do not necessarily extend to Sobolev functions on $\mathbb{R}^{2}$. Therefore

$$
\operatorname{Dom}\left(\mathrm{Ch}_{Y}\right) \supsetneq\left\{\left.f\right|_{Y^{o}} \in \mathcal{F}^{l o c}\left(Y^{o}\right): \int_{Y}\left[|D f|_{*}^{2}+f^{2}\right] d m<\infty\right\} .
$$

\subsection{Neumann heat flow as gradient flow of the entropy}

Our main goal in this section is to prove that - also on non-convex sets $Y$ - the heat flow can be uniquely characterized as gradient flow for the entropy Ent $m_{Y}$ in the space of probability measures on $Y$ equipped with the $L^{2}$-Wasserstein distance induced by $d_{Y}$.

Theorem 4.12. Let $(X, d, m)$ be a $\operatorname{RCD}^{*}(K, N)$ space and $Y$ be a regularly $\kappa$-convex set (for some $\kappa \leq 0)$ with $d_{Y}<\infty, m(Y)>0$ and $Y=\overline{Y^{o}}$. Then for all $f_{0} \in L^{2}\left(Y, m_{Y}\right)$ with $\mu_{0}=f_{0} m_{Y} \in \mathcal{P}_{2}(Y)$ the following are equivalent

(i) $t \mapsto f_{t}$ is a gradient flow for $\mathrm{Ch}_{Y}$ in $L^{2}\left(Y, m_{Y}\right)$

(ii) $t \mapsto \mu_{t}=f_{t} m_{Y}$ is a gradient flow for $\operatorname{Ent}_{m_{Y}}$ in $\left(\mathcal{P}(Y), W_{2, d_{Y}}\right)$.

In both cases, 'gradient flow' is understood in the sense of Definition 1.3

Under the additional assumption that $m(\partial Y)=0$, the heat flow on $Y$ is given in terms of the Neumann heat semigroup, see Corollary 4.9.

Let us stress once again that for non-convex $Y$ the heat flow cannot be an $\mathrm{EVI}_{l}$ heat flow for any $l$.

Our strategy to prove Theorem 4.12 relies on a number of non-trivial facts which we collect in the next section and which will now be applied to the metric measure space $\left(Y, d_{Y}, m_{Y}\right)$ in the place of $(X, d, m)$.

Proof. For each $\varepsilon>0$ let $V_{\varepsilon}$ be a regularly $\kappa$-convex function as requested in Definition 3.4 with $Y=\left\{V_{\varepsilon} \leq 0\right\}$ and $V_{\varepsilon}>-\varepsilon$ on $X$. For each positive integer $k$, set

$$
\phi_{k}(x):=e^{-2 \kappa V_{\varepsilon}(x)}
$$

where we choose $\varepsilon \in(0,1)$ so small (depending on $k$ ) that $1 \geq \phi_{k} \geq \frac{k}{k+1}$ on $Y=\left\{V_{\varepsilon} \leq 0\right\}$. Since $V_{\varepsilon}$ is in $\operatorname{TestF}(X), V_{\varepsilon}$ is bounded, so $\phi_{k} \leq c$ for some $c>0$. By construction, we have $\phi_{k}(x) \rightarrow 1$ as $k \rightarrow \infty$, for each $x \in Y$. By Theorem 2.17 (with $\kappa^{\prime}=2 \kappa$ ), $Y$ is locally geodesically convex in $\left(X, \phi_{k} \odot d\right)$.

Thus we have constructed a sequence of functions $\left(\phi_{k}\right)$ with the following properties:

(i) $1 \geq \phi_{k} \geq \frac{k}{k+1}$ on $Y$, and $\phi_{k}=1$ on $\partial Y$,

(ii) $1 \leq \phi_{k} \leq c$ on $X \backslash Y$,

(iii) For every $x \in Y, \phi_{k}(x) \rightarrow 1$ as $k \rightarrow \infty$,

(iv) $Y$ is a locally geodesically convex subset of $\left(X, \phi_{k} \odot d\right)$,

(v) $\left(X, \phi_{k} \odot d, m\right)$ satisfies $C D\left(K^{\prime}, \infty\right)$ for some $K^{\prime} \in \mathbb{R}$ that may depend on $Y, K$ and $k$. 
The last property, indeed, follows from Theorem 3.6.

For simplicity, we will write $d_{k}:=\phi_{k} \odot d$. We have

$$
\frac{k}{k+1} d_{Y}(x, y) \leq d_{k}(x, y) \leq d_{Y}(x, y) \quad \forall x, y \in Y,
$$

hence,

$$
\frac{k}{k+1} W_{2, d_{Y}}(\mu, \nu) \leq W_{2, d_{k}}(\mu, \nu) \leq W_{2, d_{Y}}(\mu, \nu) \quad \forall \mu, \nu \in \mathcal{P}(Y) .
$$

Therefore, the descending slope of the relative entropy for the Wasserstein space $\left(\mathcal{P}(Y), W_{2, d_{k}}\right)$,

$$
\left|D_{W_{2, d_{k}}}^{-} \operatorname{Ent}_{m_{Y}}(\mu)\right|:=\limsup _{\nu \rightarrow \mu, \nu \neq \mu} \frac{\left[\operatorname{Ent}_{m_{Y}}(\mu)-\operatorname{Ent}_{m_{Y}}(\nu)\right]_{+}}{W_{2, d_{k}}(\mu, \nu)},
$$

is bounded between $\left|D_{W_{2, d_{Y}}}^{-} \operatorname{Ent}_{m_{Y}}(\mu)\right|$ and $\frac{k+1}{k}\left|D_{W_{2, d_{Y}}}^{-} \operatorname{Ent}_{m_{Y}}(\mu)\right|$.

By the Convexification Theorem (Theorem 2.17), $Y$ is a locally geodesically convex subset of the $\mathrm{CD}\left(K^{\prime}, \infty\right)$-space $\left(X, d_{k}, m\right)$, hence $Y$ inherits the $\operatorname{CD}\left(K^{\prime}, \infty\right)$-condition. Thanks to Proposition 4.16, we are now in a position to apply Proposition 4.14. For $\mu=\rho m$ and $f=\sqrt{\rho}$, we obtain

$$
\begin{aligned}
4 \int_{Y}|D \sqrt{\rho}|_{*, d_{Y}}^{2} d m & \geq \liminf _{k \rightarrow \infty} 4\left(\frac{k}{k+1}\right)^{2} \int_{Y}|D \sqrt{\rho}|_{*, d_{k}}^{2} d m \\
& =\liminf _{k \rightarrow \infty}\left(\frac{k}{k+1}\right)^{2}\left|D_{W_{2, d_{k}}}^{-} \operatorname{Ent}_{m_{Y}}(\mu)\right|^{2} \\
& \geq \liminf _{k \rightarrow \infty}\left(\frac{k}{k+1}\right)^{2}\left|D_{W_{2, d_{Y}}}^{-} \operatorname{Ent}_{m_{Y}}(\mu)\right|^{2}=\left|D_{W_{2, d_{Y}}}^{-} \operatorname{Ent}_{m_{Y}}(\mu)\right|^{2} .
\end{aligned}
$$

Combining this with the opposite inequality which holds by Proposition 4.15, we get the equality

$$
4 \int_{Y}|D \sqrt{\rho}|_{*, d_{Y}}^{2} d m=\left|D_{W_{2, d_{Y}}^{-}} \operatorname{Ent}_{m_{Y}}(\mu)\right|^{2} .
$$

Finally, we apply Propositions 4.14 and 4.13 . This completes the proof of Theorem 4.12

\subsection{Facts on slopes and gradient flows}

Let a metric measure space $(X, d, m)$ be given where $(X, d)$ is a complete locally compact geodesic space and $m$ is a locally finite Borel measure with full topological support and satisfies (11). Let $\mathcal{P}(X)$ denote the space of probability measures on $X$. A measure $\mu \in \mathcal{P}(X)$ has second finite moment, denoted $\mu \in \mathcal{P}_{2}(X)$, if

$$
\int_{X} d^{2}\left(x_{0}, y\right) d \mu(y)<\infty
$$

for some (then all) $x_{0} \in X$. We say that $\mu_{n}$ converges to $\mu$ (either strongly or weakly) with moments in $\mathcal{P}_{2}(X)$ if $\mu_{n} \rightarrow \mu$ and $\int d^{2}\left(x_{0}, y\right) d \mu_{n}(y) \rightarrow \int d^{2}\left(x_{0}, y\right) d \mu(y)$ both converge (strongly or weakly, respectively).

Proposition 4.13. AGS14a, Theorem 8.5] Assume that $\left|D^{-} E n t_{m}\right|$ is sequentially lower semicontinuous w.r.t. strong convergence with moments in $\mathcal{P}_{2}(X)$ on sublevels of Ent ${ }_{m}$. Then for all $f_{0} \in L^{2}(X, m)$ such that $\mu_{0}=f_{0} m \in \mathcal{P}_{2}(X)$, the following equivalence holds:

(i) If $f_{t}$ is the gradient flow of $\mathrm{Ch}$ in $L^{2}(X, m)$ starting from $f_{0}$, then $\mu_{t}:=f_{t} m$ is the gradient flow of Ent ${ }_{m}$ in $\left(\mathcal{P}_{2}(X), W_{2}\right)$ starting from $\mu_{0}, t \mapsto \operatorname{Ent}_{m}\left(\mu_{t}\right)$ is locally absolutely continuous in $(0, \infty)$ and

$$
-\frac{\partial}{\partial t} \operatorname{Ent}_{m}\left(\mu_{t}\right)=\left|\dot{\mu}_{t}\right|^{2}=\left|D^{-} \operatorname{Ent}_{m}\left(\mu_{t}\right)\right|^{2} \quad \text { for a.e. } t \in(0, \infty) .
$$


(ii) Conversely, if $\mid D^{-}$Ent $t_{m} \mid$ is an upper gradient of Ent $t_{m}$, and $\mu_{t}$ is the gradient flow of Ent $_{m}$ in $\left(\mathcal{P}_{2}(X), W_{2}\right)$ starting from $\mu_{0}$, then $\mu_{t}=f_{t} m$ and $f_{t}$ is the gradient flow of $\mathrm{Ch}$ in $L^{2}(X, m)$ starting from $f_{0}$.

Proposition 4.14. AGS14a, Theorem 7.6] $\mid D^{-}$Ent $t_{m} \mid$ is sequentially lower semicontinuous w.r.t. strong convergence with moments in $\mathcal{P}_{2}(X)$ on sublevels of Ent $t_{m}$ if and only if

$$
4 \int_{X}|D \sqrt{\rho}|_{*}^{2} d m=\left|D^{-} \operatorname{Ent}_{m}\right|^{2}(\mu), \quad \forall \mu=\rho m \in \operatorname{Dom}\left(\operatorname{Ent}_{m}\right) .
$$

Proposition 4.15. [AGS14a, Theorem 7.4] Let $\mu=\rho m \in \operatorname{Dom}\left(E n t_{m}\right)$ with $\mid D^{-}$Ent $_{m} \mid(\mu)<\infty$. Then $\sqrt{\rho} \in \operatorname{Dom}\left(\mathrm{Ch}_{*}\right)$ and

$$
4 \int_{X}|D \sqrt{\rho}|_{*}^{2} d m \leq\left|D^{-} \operatorname{Ent}_{m}\right|^{2}(\mu)
$$

Proposition 4.16. AGS14a, Proposition 9.7] If $C D(K, \infty)$ holds, then $\mid D^{-}$Ent $_{m} \mid$ is sequentially lower semicontinuous w.r.t. weak convergence (hence strong convergence) with moments in $\mathcal{P}_{2}(X)$ on sublevels of Ent $t_{m}$. In particular, the gradient flow of the relative entropy can be identified with the heat flow in the sense of Proposition 4.13 .

\section{References}

[AB03] Stephanie Alexander and Richard L. Bishop. FK-convex functions on metric spaces. Manuscripta Math. 110:115-133, 2003.

[AGS14a] Luigi Ambrosio, Nicola Gigli, and Giuseppe Savaré. Calculus and heat flow in metric measure spaces and applications to spaces with Ricci bounds from below. Invent. Math., 195(2):289-391, 2014

[AGS05] Luigi Ambrosio, Nicola Gigli, and Giuseppe Savaré. Gradient flows in metric spaces and in the space of probability measures. Lectures in Mathematics ETH Zürich. Birkhäuser Verlag, Basel, 2005.

[AGS14b] Luigi Ambrosio, Nicola Gigli, and Giuseppe Savaré. Metric measure spaces with Riemannian Ricci curvature bounded from below. Duke Math. J., 163(7):1405-1490, 2014.

[AGS15] Luigi Ambrosio, Nicola Gigli, and Giuseppe Savaré. Bakry-Émery curvature-dimension condition and Riemannian Ricci curvature bounds. Ann. Probab., 43(1):339-404, 2015.

[AS96] Luigi Ambrosio and Halil Mete Soner. Level set approach to mean curvature flow in arbitrary codimension. J. Differential Geom., 43(4):693-737, 1996.

[BS10] Kathrin Bacher and Karl-Theodor Sturm. Localization and tensorization properties of the curvature-dimension condition for metric measure spaces. J. Funct. Anal., $259(1): 28-56,2010$.

[CL12] Der-Chen Chang and Yutian Li. SubRiemannian geodesics in the Grushin plane. $J$. Geom. Anal. 22:800826, 2012.

[Che99] J. Cheeger. Differentiability of Lipschitz functions on metric measure spaces. Geom. Funct. Anal., 9(3):428-517, 1999.

[EKS15] Matthias Erbar, Kazumasa Kuwada, and Karl-Theodor Sturm. On the equivalence of the entropic curvature-dimension condition and Bochner's inequality on metric measure spaces. Invent. Math., 201(3):993-1071, 2015.

[Gig] Nicola Gigli. Nonsmooth differential geometry. arXiv:1407.0809.

[HM] Bang-Xian Han, Anna Mkrtchyan. Conformal transformation on metric measure spaces. arXiv:1511.03115v2. 
[HK98] Juha Heinonen and Pekka Koskela. Quasiconformal maps in metric spaces with controlled geometry. Acta Math., 181(1):1-61, 1998.

[JKO98] Richard Jordan, David Kinderlehrer, Felix Otto. The variational formulation of the Fokker-Planck equation. SIAM J. Math. Anal. 29, 29(1): 1-17, 1998.

[Ket15] Christian Ketterer. Obata's rigidity theorem for metric measure spaces. Anal. Geom. Metr. Spaces, 3:278-295, 2015.

[LV09] John Lott and Cedric Villani, Ricci curvature for metric-measure spaces via optimal transport, Annals of Math. 169: 903-991, 2009.

[Maz11] Vladimir Maz'ya, Sobolev spaces with applications to elliptic partial differential equations, Grundlehren der Mathematischen Wissenschaften, Springer, Heidelberg 342:xxviii+866, 2011.

[Sha00] Nageswari Shanmugalingam. Newtonian spaces: an extension of Sobolev spaces to metric measure spaces. Rev. Mat. Iberoamericana, 16(2):243-279, 2000.

[Sil74] Martin L. Silverstein. Symmetric Markov processes. Lecture Notes in Mathematics, Vol. 426. Springer-Verlag, Berlin-New York, 1974.

[Stu] Karl-Theodor Sturm. Gradient flows for semiconvex functions on metric measure spaces existence, uniqueness and Lipschitz continuity. arXiv:1410.3966v2.

[Stu06] Karl-Theodor Sturm. On the geometry of metric measure spaces. I. Acta Math., 196(1):65-131, 2006.

[Vil09] Cédric Villani. Optimal transport, volume 338 of Grundlehren der Mathematischen Wissenschaften [Fundamental Principles of Mathematical Sciences]. Springer-Verlag, Berlin, 2009. Old and new. 